\title{
Look Again, and Not Just at Behavioural and Cognitive Psychotherapy
}

Take a look at our circulation and impact! As a journal we are high on circulation (about 10000 print copies including those to members, and an additional very substantial on-line readership). Our impact factor, which reflects the extent of attention our articles get in terms of citations by researchers worldwide, has been modest but steadily increasing. We are pleased to announce a further improvement in our Impact factor, from a healthy 1.491 in the previous year to its current 1.692. The Journal has also climbed 10 places in its subject category (Psychology, Clinical) and is now ranked higher than Cognitive Therapy and Research. This places us in an excellent position in the field. This is extremely good news, as it means that we are even more likely to be a target for high quality articles with the combination of good academic quality and clinical relevance.

I notice that some other journals, notably Behaviour Research and Therapy and Journal of Anxiety Disorders will, as a matter of policy, no longer consider papers that include a major component of scale/questionnaire development. Please note that Behavioural and Cognitive Psychotherapy and our sister journal, the Cognitive Behaviour Therapist, are very happy to look at such submissions, both in terms of the development of clinically useful scales and in studies where the absence of measures requires the development and validation of an existing measure in order to investigate particular hypotheses or issues.

Which brings me to the complementary role we have as a journal. As Editor of Behavioural and Cognitive Psychotherapy, the BABCP journal with a more academic and applied science emphasis, I am delighted at the way in which our sister journal, the Cognitive Behaviour Therapist, has developed and grown under the expert guidance of Michael Townend and his editorial team. Having established the journal, Michael is stepping down in 2013, with a successor yet to be decided. We are all grateful to Michael for the solid foundations he has established. Readers and contributors to Behavioural and Cognitive Psychotherapy would do well to look at how the Cognitive Behaviour Therapist has developed.

We have been working alongside $t C B T$ for a while now, and wish to take this opportunity to announce closer integration of submissions. At present, Associate Editors for Behavioural and Cognitive Psychotherapy can choose to recommend resubmission of a manuscript not suitable for $B C P$ to $t C B T$, which requires authors to begin the submission process again. This informal re-submission channel will henceforth be streamlined for those who indicate that they wish to be part of a revised submission process for $B C P$, with authors being asked at submission to decide whether they wish their submissions to be considered for $t C B T$, and to allow transfer of the manuscript including, as appropriate, transmission of reviewers' comments (at the discretion of the $B C P$ Associate Editor) where this seems likely to facilitate manuscript handling. We will add an additional decision option to $B C P$ manuscript handling, that of consideration by $t C B T$ editors. This means that, in effect, those who choose to do so can submit to both journals sequentially. 
So, to remind both readers and those who are considering submitting articles, the Cognitive Behaviour Therapist is a fully peer reviewed online journal aimed primarily at cognitive behavioural practitioners. It features papers covering clinical and professional issues, which contribute to the theory, practice and evolution of the cognitive and behavioural therapies. It publishes papers that describe new developments and articles that are practice-focused, including those that detail clinical interventions. It also publishes research reports concerning the practice of cognitive behaviour therapy, from traditional research designs through to detailed case reports and audits that are relevant to practice. It will also publish reviews of clinical scales and papers that have an education, training or supervision focus. Reviews and integrations of previously published literature directly relevant to practitioners will also be accepted. A particular feature of the journal is that its electronic nature is designed to ensure timeliness of publication and professional debate whilst also ensuring rigorous standards in the dissemination of high-quality material with relevance to the practice of the cognitive and behavioural therapies. Over the next few months, online responses and discussion will be incorporated. If you haven't already done so, you may want to look at it here: www.journals.cambridge.org/CBT

To access the journal free, a BABCP member will need to first register their details and choose a username and password; once registered and logged in, the member can then "activate subscriptions" by entering their membership number and BABCP society ID number, 71650, into the appropriate fields. Back issues of the journal are then available for access on line and articles can be downloaded.

We would like to express our gratitude to two groups of people. First, since the last editorial, several Associate Editors have stepped down, including Adrian Wells, David Westbrook, Sam Cartwright-Hatton and Ian James. I would like to thank them on behalf of myself, the BABCP and the rest of the editorial team for the work that they have carried out over the last few years. Next, I would like to welcome the new Associate Editors: these are Peter Langdon, Rebecca Murphy, Kate Rimes, Ailsa Russell, Paul Stallard and Kim Wright. Welcome!

Finally, this is the last issue of the journal with the current cover. Watch out for the first issue of 2013 which will bring a new look!

Paul Salkovskis

Editor, Behavioural and Cognitive Psychotherapy 